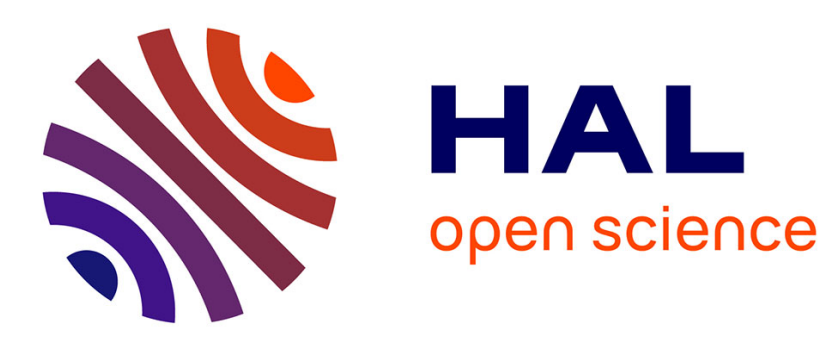

\title{
Champ du regard et surgissement de la présence dans les processus créateurs
}

Sylvie Le Poulichet

\section{To cite this version:}

Sylvie Le Poulichet. Champ du regard et surgissement de la présence dans les processus créateurs. Cliniques méditerranéennes, 2010, L'imposture dans le siècle, 1 (81), pp. 259 - 270. 10.3917/cm.081.0259 . hal-01513899

\section{HAL Id: hal-01513899 \\ https://hal.science/hal-01513899}

Submitted on 25 Apr 2017

HAL is a multi-disciplinary open access archive for the deposit and dissemination of scientific research documents, whether they are published or not. The documents may come from teaching and research institutions in France or abroad, or from public or private research centers.
L'archive ouverte pluridisciplinaire HAL, est destinée au dépôt et à la diffusion de documents scientifiques de niveau recherche, publiés ou non, émanant des établissements d'enseignement et de recherche français ou étrangers, des laboratoires publics ou privés. 


\section{CHAMP DU REGARD ET SURGISSEMENT DE LA PRÉSENCE DANS LES PROCESSUS CRÉATEURS}

Sylvie Le Poulichet

\section{ERES | «Cliniques méditerranéennes »}

2010/1 n 81 | pages 259 à 270

ISSN 0762-7491

ISBN 9782749212432

Article disponible en ligne à l'adresse :

http://www.cairn.info/revue-cliniques-mediterraneennes-2010-1-page-259.htm

\section{Pour citer cet article :}

Sylvie Le Poulichet, « Champ du regard et surgissement de la présence dans les processus créateurs », Cliniques méditerranéennes 2010/1 (n81), p. 259-270. DOI 10.3917/cm.081.0259

Distribution électronique Cairn.info pour ERES.

(C) ERES. Tous droits réservés pour tous pays.

La reproduction ou représentation de cet article, notamment par photocopie, n'est autorisée que dans les limites des conditions générales d'utilisation du site ou, le cas échéant, des conditions générales de la licence souscrite par votre établissement. Toute autre reproduction ou représentation, en tout ou partie, sous quelque forme et de quelque manière que ce soit, est interdite sauf accord préalable et écrit de l'éditeur, en dehors des cas prévus par la législation en vigueur en France. Il est précisé que son stockage dans une base de données est également interdit. 


\title{
Sylvie Le Poulichet
}

\author{
Champ du regard \\ et surgissement de la présence \\ dans les processus créateurs
}

En se référant essentiellement à des œuvres littéraires, Freud pouvait penser que certains créateurs témoignent d'une "profonde connaissance des processus psychiques ${ }^{1} »$. Et la pertinence de cette remarque datant de 1907 n'allait pas être démentie par la suite. Encore faut-il préciser à ce propos qu'une " profonde connaissance » ne se laisse pas nécessairement assimiler à l'exercice d'un savoir conscient donnant lieu à une description littéraire. Les expériences de plasticiens et de musiciens, entre autres, nous livrent des éléments précieux concernant des processus psychiques que la théorie classique de « la sublimation » n'avait pu approcher.

Plus précisément, ce qui n'était pas possible en 1907 et qui l'est devenu aujourd'hui serait bien la prise en considération de l'apport des créateurs qui se prêtent à l'expérience analytique. Il se trouve que la clinique nous offre les occasions de relever en bien des cas l'originalité de ces cures. La spécificité des questions qui s'y trouvent soulevées et les effets qu'elles visent, parallèlement et au-delà des processus habituellement mis en jeu dans les cures analytiques, apportent-ils un nouvel éclairage sur la dynamique psychique?

Concernant le style des questionnements déployés dans ces analyses, les formules de trois questions soulevées par ces patients s'imposent d'emblée, de par leur caractère suffisamment crucial et général tout à la fois : comment prendre corps et faire surgir la présence ? Comment faire tenir un être au monde en créant dans le plaisir et non dans la souffrance ? Et encore :

Sylvie Le Poulichet, psychanalyste, professeur de psychopathologie à l'université Paris 7-Denis-Diderot ; 4 rue Dareau F-75014 Paris.

1. S. Freud (séance du 4 décembre 1907), Les premiers psychanalystes. Minutes de la Société psychanalytique de Vienne, tome 1, Paris, Gallimard, 1976, p. 273. 
comment consentir au désir et à ce qui arrive, y compris en un lien possible à l'autre?

L'activité créatrice, manifestant souvent ses prémices depuis l'enfance, constituait déjà bien sûr une manière d'apporter des réponses à certaines de ces questions, même si ces dernières ne pouvaient alors être formulées avec autant de précision. Néanmoins subsistait pour beaucoup un « empêchement $^{2}$ », qu'il est toujours particulièrement émouvant de voir céder au cours du processus analytique, pour ceux qui ont choisi de s'y engager pleinement.

Il faut alors soulever ici une autre question : en quoi le processus analytique est-il susceptible d'amplifier les modalités de " prise de corps », de «surgissement de la présence » et de relance du désir que visait déjà l'activité créatrice?

\section{SURGIR EN UNE PRISE DE CORPS}

S'inspirant des travaux de Merleau-Ponty, Lacan évoquait en 1964 « ce regard qui nous cerne, et qui fait d'abord de nous des êtres regardés ${ }^{3}$ ». Il relevait alors combien cette fonction du regard, différenciée de celle de l'œil, se trouve particulièrement mise en évidence dans le champ du rêve où se manifestent " l'absence d'horizon, la fermeture, de ce qui est contemplé dans l'état de veille, et, aussi bien, le caractère d'émergence, de contraste, de tache, de ses images, l'intensification de leurs couleurs ${ }^{4} »$. C'est ainsi qu'apparaît « ce qui nous cerne », le plus souvent à notre insu. Moins insu peut-être pour de nombreux créateurs qui appellent chaque jour ce " cerne » conférant ses bords à l'existence.

Dans le champ de la création, ce qui nous regarde, ce qui nous cerne, par émergence de contrastes et intensification de couleurs, aurait le pouvoir de faire surgir la présence associée à une sensation d'épaisseur charnelle. Surgissement de la présence et " prise de corps » sont bien ici corrélatifs : la montée du regard qui cerne, qui tapisse l'horizon, donne lieu au corps en tant qu'émergence sensible. C'est ce que je désigne comme une "prise de corps ", qui ne se réduit pas à la vision de l'image du corps dans un miroir.

D'une manière générale, la prise de corps est un surgissement privilégié du corps pulsionnel investi par les images et le langage. Plus précisément, cette prise de corps s'accomplit lorsque ce dernier est saisi dans un champ

2. En référence au texte de Samuel Beckett : «Peintres de l'empêchement », dans Le monde et le pantalon, Paris, Éd. Minuit, 1989.

3. J. Lacan, Le Séminaire, Livre XI, Les quatre concepts fondamentaux de la psychanalyse, Paris, Le Seuil, 1973, p. 71.

4. Ibid., p. 72. 
de regard et de désir, lorsqu'il est regardé plus qu'il ne voit, comme dans l'expérience du stade du miroir où l'image acquiert de la profondeur grâce au regard désirant et à la parole de l'autre. Nous pouvons d'ailleurs penser qu'en ce stade, une vision de l'image du corps propre qui ne serait pas portée par un champ de regard et de désir venant du lieu de l'Autre, ne pourrait engendrer une véritable prise de corps, et qu'elle se réduirait à une identification à une "statue » figée ${ }^{5}$. Néanmoins, ultérieurement, l'événement d'une prise de corps n'implique pas nécessairement la présence et la voix de l'autre, comme en témoigne le pouvoir d'incarnation que figurent l'art et le rêve.

Et l'on entend dans les cures de nombreux créateurs - plus explicitement chez des peintres - les mises en jeu et en question permanente de cette prise de corps équivalente à l'émergence d'un champ de regard qui secrète l'événement de la présence. La fonction de l'être cerné, inhérente à l'être regardé, ne saurait être réduite à celle d'une enveloppe ou d'une ligne qui délimite, ni à celle d'une surface " pare-excitations » qui protège de l'effraction. Car il s'agit plutôt de se trouver transpercé ou submergé par les choses, comme le relève Merleau-Ponty :

« Entre lui et le visible, les rôles inévitablement s'inversent. C'est pourquoi tant de peintres ont dit que les choses les regardent, et André Marchand après Klee : "Dans une forêt, j'ai senti à plusieurs reprises que ce n'était pas moi qui regardais la forêt. J'ai senti, certains jours, que $c^{\prime}$ étaient les arbres qui me regardaient, qui me parlaient... Moi j'étais là, écoutant... Je crois que le peintre doit être transpercé par l'univers et non vouloir le transpercer... J'attends d'être intérieurement submergé, enseveli. Je peins peut-être pour surgir ${ }^{6} . "$ ”

Entrer ainsi dans un champ de regard afin de surgir, afin d'être ponctuellement la présence en tant que surgissement après avoir été submergé : c'est en effet ce qu'expriment de diverses manières de nombreux créateurs, aussi bien musiciens que peintres. Merleau-Ponty écrit aussi : " Le monde n'est plus devant lui par représentation : c'est plutôt le peintre qui naît dans les choses comme par concentration et venue à soi du visible ${ }^{7} . .$. » On pourrait aussi bien évoquer ici la venue à soi du sonore, et dans les deux cas s'accomplit en effet une subversion de l'ordre et du sujet de la représentation.

La « prise de corps » s'effectue paradoxalement en ce champ de regard où « je » est submergé, transpercé : c'est cela qui ouvre la possibilité du geste

5. Selon l'expression de J. Lacan dans « Le stade du miroir comme formateur de la fonction du Je » (1949), dans Écrits, Paris, Le Seuil, 1966, p. 95.

6. M. Merleau-Ponty, « L'œil et l'esprit », Les temps modernes n 184-185, 1961, p. 202.

7. Ibid., p. 217. 
créateur qui permet de surgir. En cette "prise », il ne s'agit donc pas d'avoir ou de posséder un corps opérant, mais bien plutôt d'être le jaune, d'être le bleu, comme dans le champ du rêve où l'on ne voit pas, mais où l'on est le papillon dont on rêve, " papillon pour personne " précise Lacan se référant au rêve de Tchouang-Tseu. Et Lacan d'ajouter que " c'est quand il était le papillon qu'il se saisissait à quelque racine de son identité - qu'il était, et qu'il est dans son essence, ce papillon qui se peint à ses propres couleurs - et c'est par là, en dernière racine, qu'il est Tchouang-Tseu ${ }^{8}$ », alors que " $c^{\prime}$ est quand il est éveillé qu'il est Tchouang-Tseu pour les autres et qu'il est pris dans leur filet à papillons ${ }^{9} »$.

Cette homologie, entre les champs de regard dans le rêve et dans l'activité créatrice appartenant au monde de la veille, n'est que partielle : être le geste vers la toile, être le jaune, être la surface de contact entre les cordes de l'archet et celles du violon, n'est pas tout à fait identique au fait d'être le papillon dont on rêve. Néanmoins ils engagent chacun des modalités de devenir-autre pour personne à travers d'insolites prises de corps, même si le rêve sera éventuellement relaté ultérieurement à un analyste, même si les compositions musicales ou picturales seront éventuellement jouées ou exposées ultérieurement pour un public.

Le corps passagèrement saisi en un champ de regard et de présence ne se voit pas lui-même de l'extérieur et il ne met pas en perspective l'image d'un moi idéal narcissique. Cependant, les deux dimensions peuvent souvent entrer en conflit, tout particulièrement chez les créateurs pour lesquels la représentation d'un idéal inaccessible se noue à celle d'un interdit intériorisé qui prescrit l'échec, l'inhibition ou le renoncement. De ce point de vue, l'un des enjeux de la cure analytique consiste à dénouer fil à fil les prescriptions mortifères et les identifications aliénantes associées à l'interdit et à l'idéal, afin de laisser toute sa puissance au champ de désir et de regard déployé dans la présence.

Le fait de prendre corps en un champ de regard qui fait surgir la présence constitue en effet la condition première au geste de création, alors que ceux qui ont très tôt accédé et adhéré à une vision normalisante du miroir n'ont pas été envahis par la nécessité de faire éclore cette présence pour l'habiter. A contrario, pour ceux qui n'ont pu que se détourner partiellement d'un miroir déformant car déformé par la voix et le regard mortifères d'un Autre primordial, il est nécessaire de se déplacer et d'inventer d'autres surfaces de résonance ou d'autres volumes donnant prise au corps. Et qu'entendre en ce « je peins pour surgir » ? Certainement pas la visée de l'affirmation ou de la représentation d'un moi devant autrui. Il s'agit plutôt de la dimension de

8. J. Lacan, Le Séminaire, Livre XI..., op. cit., p. 72.

9. Ibid., p. 73. 
l'émergence d'un éclat de présence que l'on pourrait qualifier d'aveugle, dès lors qu'un champ de regard lui donne sa consistance.

Le rêveur ne voit pas, " il suit » dit Lacan. De même, dans le moment créateur, le sujet ne voit rien, il est submergé par une intensité qui le fait surgir.

\section{UNE POUSSÉE POÉTIQUE QUI COUPE LE SOUFFLE}

À propos de la dernière grande toile qu'elle a réalisée pour une commande publique, une analysante dit :

«Le corps sait ce qu'il faut faire, j'ai lâché le contrôle. Le flux ne fait que passer par moi, ce n'est pas à moi, je ne suis qu'un passage. Je m'aveugle pour peindre. Il n'y a rien à rater ni à réussir, mais seulement à désirer. [...] La puissance du sentiment d'exister est très présente quand l'être est plutôt en expansion, envahi par un flux. Ce n'est pas lié à la personne. Je suis en accord avec la musique de ce motif et ces mobiles que je découvre en les créant. Cela fait vingt ans que j'essayais de m'approcher de ça. »

Avant l'analyse, dit-elle, sa manière de peindre ressemblait à une obsession de laisser des traces, qui empêchait partiellement la présence. Elle était obligée de réaliser de grandes choses difficiles et d'être déçue par elle-même. Avant que le " processus de dressage et de contrôle » ne cesse, il lui fallait se couper du monde pour souffrir seule avec la peinture. Elle voulait maintenant « peindre autrement en faisant corps avec le monde ».

Pourquoi se couper du monde à cause de la peinture ou faire corps avec lui grâce à la peinture ? L'analysante précise que la peinture est pour elle « le lieu de passage entre mort et vivant » et que «la définition de peindre, c'est juste d'être présente, en mouvement et vouloir de l'instant». Et elle ajoute : " Je suis moins dans les limbes. Avant j'existais pour faire don d'organe ou de nourriture. J'en ai assez d'être une ombre, je veux avoir un corps. »

Quitter les limbes, avoir un corps et pratiquer la peinture comme lieu de passage entre le mort et le vivant, prenait un sens particulier pour cette analysante qui s'était sentie très tôt assignée à une place insolite : "Quand ma mère était enceinte de moi, son frère peintre s'est tiré une balle dans la tête. J'ai l'impression que ma mère n'est jamais sortie de son deuil. »

Elle se revoit alors, enfant, faisant « surgir des espaces habitables avec le dessin et le modelage : c'était comme une création de moi-même ». Ce qui l'envahissait déjà était l'incertitude d'être née, tandis qu'elle devait pour ses parents « répondre subitement à l'exigence d'incarner telle ou telle forme censée donner le spectacle de la vie ». L'expression de ses propres 
émotions ou sentiments était en quelque sorte disqualifiée ou interdite par ses parents.

Le devoir de peindre qui l'écrasait, de même que l'obligation d'effacer pour " faire toujours mieux » lui rappellent également l'enfance : "J'étais habituée à tellement d'impossible qu'il fallait fabriquer des objets, fabriquer de l'art pour me décaler. C'était une nécessité vitale face à une mère jamais satisfaite, d'une grande violence, experte en souffrance et en sacrifice, si bien qu'on ne pouvait jamais l'atteindre. » Ce nécessaire décalage, ce " pas de côté » ou ce déplacement qui amènent à créer des tenant-lieu du moi se retrouvent chez de nombreux créateurs, qui évitent ainsi de se trouver entièrement aspirés dans la tentative d'offrir leur propre moi ou leur propre corps comme objet susceptible de combler l'autre.

L'analysante questionnait : «Comment cohabiter de manière heureuse avec une poussée poétique qui coupe le souffle depuis l'enfance ? Je sentais aussi que j'étais un pays colonisé, occupé par une puissance extérieure. J'habitais dans la cave de moi-même, le reste était occupé. » La poussée poétique coupe le souffle car " penser, c'est dans le corps ». Auparavant, cela impliquait de rester dans une solitude destructrice et dans le désamour. Alors qu'aujourd'hui, cette " poussée » se manifestait à travers les « surgissements de nouvelles strates de désir », avec l'impression de découvrir que son univers était recevable, dans le jeu, l'énigme et le plaisir. Et, dit-elle, " moi-même a explosé, ce qui voulait à tout prix être reconnu : j'ai réussi à jeter dehors une personne qui était en moi ». Ainsi s'engageait une décolonisation.

C'est là l'une des batailles qui fut livrée et gagnée en analyse : au-delà de la mère qui semblait se nourrir du non-désir, se trouvait un père tyrannique dont il a fallu dire lors d'une séance " qu'il était mort désormais ». Faire advenir la dimension du père mort devenait en effet une condition de survie. Ce père, qui se trompait sur son âge et qui entrait dans des colères folles lorsqu'elle osait rappeler sa propre date de naissance, lui semblait avoir toujours signifié : «Tu n'es pas née ! » Et il avait fallu sortir de l'ornière de cette terreur en laquelle le père pouvait à tout moment signifier « Je te retire la vie » (ce qui pouvait entre autres s'accompagner de l'exhibition d'un fusil de chasse). Cependant, l'analysante savait désormais que la figure la plus dangereuse était celle du père intériorisé, et elle ajoutait : «Il ne faut pas que je me fasse disparaître, comme mon père me faisait disparaître. »

L'analysante sentait aussi à quel point elle avait tenté d'incarner la fille idéale dont rêvait ce père, mais que le simple fait d'exister entrait en contradiction avec ce rêve. Elle avait pu en analyse chasser ce père intériorisé qu'elle décrivait comme " un vampire »: " Il n'a pas de reflet ni d'énergie propres, il faut qu'il les vole, qu'il détruise, qu'il contrôle et salisse. » Des rêves avaient mis en images ce vol, ce contrôle, cette destruction. De plus, la 
figure du vampire sans image dans le miroir l'avait amenée à dire sa propre difficulté à s'autoriser à être visible : "Si je suis visible, ça veut dire que je laisse tomber le père. Comment faire pour entrer dans mon univers, pour faire surface en me passant de la validation du père ? »

«Faire surface » avait été, au sens propre, une solution pratiquée depuis l'enfance : elle avait toujours dessiné ce qui l'entourait pour donner aux choses et aux êtres leurs images, comme si seuls les traits de crayon pouvaient leur conférer une véritable existence. Elle semblait ne s'être jamais sentie véritablement regardée, tandis qu'elle se faisait pourvoyeuse de reflet, surtout pour le père. N'avait-elle pas annoncé dès le début de l'analyse qu'elle ne voulait plus être une ombre, qu'elle souhaitait maintenant avoir un corps?

Avec la dernière grande toile qu'elle venait de réaliser se recomposait autrement le besoin de fabriquer des images : "La peinture était une preuve de mon existence. Là, si je rate, je vais continuer à exister. Une longue dépression infantile est en train de finir. Cela se joue dans la couleur dense, saturée, vive. Il y a une grande joie et de la confiance. » N'était-elle pas devenue "papillon qui se peint à ses propres couleurs ", qui se peint au désir de son propre rêve, au lieu d'incarner l'image dont rêve le père ? N'était-elle pas devenue celle qui parle à ses propres images, au lieu de se trouver réduite à un reflet du père sans visage à force d'user de voix contradictoires ?

Pourquoi une telle mutation, qui concernait aussi sa capacité d'aimer, avait-elle ainsi eu lieu dans l'analyse ? Il apparaît clairement qu'un véritable changement psychique s'était accompli lorsque des gestes de parole - de l'analysante et de l'analyste - avaient en quelque sorte mis en scène, en sens et en images un meurtre symbolique de la figure du père tyrannique, rendant caduque l'ancienne culpabilité de l'analysante. Et il faut bien admettre que cela engage entre autres la capacité de l'analyste à assumer une heureuse complicité autour d'un meurtre symbolique (ce qui peut surprendre, puis soulager considérablement un analysant).

Parallèlement à l'élaboration autour des figures parentales, associée à ce que cette analysante nommait « la confiance éprouvée en analyse », permettant un " lâcher prise ", s'était produit un événement dans le transfert : l'entrée dans un nouveau champ de regard au fil des séances. Alors même qu'allongée sur le divan, elle n'était pas visible pour l'analyste, montaient un champ de regard et une densité de présence dans la résonance des paroles et du silence. Entre autres événements, c'est à l'occasion de doutes qu'elle avait un jour exprimés concernant son avenir immédiat et la tentation d'interrompre toutes ses activités que je lui avais formulé : "Il y a une partie de vous qui sait où est votre désir ", au lieu d'intervenir directement face à l'annonce d'un passage à l'acte possible. Et elle avait énoncé à la séance 
suivante combien cette parole était venue comme un événement surprenant lui donner une heureuse nouvelle. Elle avait ajouté : «Je ne peux plus faire comme si je ne savais pas, il y a un fil et non une zone blanche sur la carte. Je ne veux plus être envahie par les autres. »C'est notamment à partir de ce moment-là qu'un nouveau champ de regard semblait s'être ouvert, comme si les lieux du savoir et du voir convergeaient vers elle, la regardaient. Elle était désormais regardée par la parole, cernée par un champ de désir qui lui donnait corps, au lieu de « se laisser tomber » comme avant l'analyse.

En conséquence, tout un processus se déployait, qui lui permettait de commencer à répondre à sa propre question si fine, si pertinente : «Comment parler en images aux images que l'on porte ? " Car seul ce " parler en images » était supposé pouvoir les éveiller, les remettre en mouvement, les saisir là où elles se trouvaient et les transformer, dans le temps même où « le corps entier " s'engageait dans un travail avec la couleur.

\section{DISPOSITIF SPÉCULAIRE TRANSFÉRENTIEL ET FABRIQUE DU LIEU}

De manière plus générale, il apparaît qu'un dispositif spéculaire transférentiel peut s'élaborer dans la cure, de telle sorte que des images parlent à d'autres images en un dispositif où la parole génère une forme d'habitacle corporel et un sentiment de continuité d'existence. Le champ du transfert et les surfaces de réflexion constitués par le rapport au lieu de l'Autre engendrent une mise en mouvement des images en lesquelles prennent consistance des signifiants. Les surfaces de réflexion et la spécularité que façonne le transfert ne sont pas assimilables aux effets d'un miroir plan immobile. Elles renvoient davantage à la métaphore de la pierre spéculaire : minéral qui peut se diviser en feuillets minces, transparents et capables de réfléchir la lumière. Il ne s'agit pas d'un jeu de miroir entre l'analyste et l'analysant, mais davantage de l'émergence d'un champ de regard et de présence qui rend les images parlantes et donne lieu à des paroles ancrées dans une corporéité.

Au moment même où tout ce dispositif spéculaire transférentiel ${ }^{10} 1^{\prime}$ amenait à ne plus s'effacer, à ne plus effacer ses propres images et à entrer en un nouveau champ de regard, l'analysante créait pour un espace public une fresque en laquelle un grand miroir et des projecteurs allaient cerner la très grande toile qu' elle avait réalisée. L'ombre et la lumière portées, ainsi que le reflet du miroir, devaient converger vers la toile. Il s' agissait en quelque sorte de la fabrication d'un immense appareil optique, dont elle ne savait pas, jusqu'aux derniers ajustements techniques, s'il allait vraiment fonctionner.

10. Notion que j'avais présentée sans y adjoindre ces précisions dans mon ouvrage Psychanalyse de l'informe. Dépersonnalisations, addictions, traumatismes, Paris, Éd. Aubier/Flammarion, 2003, p. $45-57$. 
Les corps des usagers qui passaient en cet endroit devaient être reflétés par le miroir et s'inscrire en ce dispositif. Il fallait que se produise là un véritable " fait pictural » qui ne se réduise pas à un effet et à un but décoratifs. C'est bien cela qui s'appelait " créer une image » : faire surgir un espace où l'on peut prendre corps, c'est-à-dire porter la fabrique d'un lieu.

Comme on l'a vu, la fabrique d'un lieu du corps, tel un dispositif déployé en un champ de regard et de présence, n'avait pu ici s'élaborer véritablement qu'à la condition que soit produit au préalable en analyse l'accomplissement d'un heureux geste symbolique meurtrier, à savoir l'équivalent d'un meurtre de la figure du père tyrannique (qui peut très bien en certains cas renvoyer aussi à un personnage maternel...). La fabrique du lieu et le meurtre symbolique ne représentent pas là de simples actes ponctuels, mais bien des événements qui modifient durablement l'économie des processus psychiques. De manière plus générale, un tel lieu du corps peut surgir lorsque se transforment des identifications et des investissements conflictuels, ou lorsque se dénoue une identification mélancolique partielle, même si cela ne coïncide pas toujours exactement avec la création d'une œuvre aussi particulière.

\section{LA VOLUMINOSITÉ}

En ces cures, il apparaît essentiel de ne pas dissocier ce qui s'élabore en séance et ce qui œuvre à " l'atelier » (de peinture, de musique ou d'écriture...), car le dispositif spéculaire transférentiel qui se construit dans les séances dialogue en permanence avec ce qui surgit à «l'atelier», convergeant ensemble vers une nouvelle prise de corps ancrée dans la présence. Dans ce contexte, chaque cure analytique amène plus que jamais le psychanalyste à se laisser surprendre par de nouveaux modes de composition et de recomposition de la réalité psychique, redéployant le champ des images où s'inscrivent les traits symboliques fondateurs d'une origine et d'un à-venir. Aucun savoir constitué - y compris la théorie freudienne de la sublimation - ne saurait se trouver « appliqué » à ces cures, car elles y perdraient en quelque sorte la force de leur dynamique propre et la dimension d'émergence de l'inconnu qui constituent leurs ressorts.

Chacune de ces cures suscite son propre dispositif spéculaire transférentiel, entrant en résonance avec des fabriques de lieux et des "processus d'émergence d'objets inconnus ${ }^{11}$ » qui captent des forces pulsionnelles en devenir. De tels processus appellent encore leur propre métamorphose et leur recomposition en d'autres fabriques de lieux, sans épuiser ni obturer la question du désir.

11. Selon l'expression que j'utilisais dans mon ouvrage L'art du danger. De la détresse à la création, Paris, Éd. Anthropos, 1996, p. 1-9. 
En cette clinique peut résonner particulièrement la proposition freudienne selon laquelle le moi serait " avant tout un moi corporel », " projection d'une surface ${ }^{12}$ ", car à travers la création soutenue par le processus analytique, une surface corporelle événementielle semble bien constituer une fabrique de lieu du corps se matérialisant aussi hors du corps propre. Ainsi $\mathrm{s}^{\prime}$ entendent les « processus d'engendrement de corps étrangers ${ }^{13}$ » qui décentrent le moi et ce que l'on nomme l'investissement narcissique. Plus particulièrement, une manière de précipiter le corps entier dans la fabrique du lieu laisse entendre la nécessité pour de nombreux créateurs de prendre corps et présence à travers ce saut ou ce geste qui en certains cas préservent d'un anéantissement, ou qui en d'autres cas incarnent une manière de mordre sur la réalité, même si l'élaboration du lien à l'autre accorde aussi parallèlement un nouveau lieu d'ancrage.

Faut-il d'ailleurs se contenter d'une référence à la " projection d'une surface corporelle » lorsque des substituts du moi incarnent des fabriques de lieux à travers le geste créateur ? Une prise de corps, dans le champ de la création, se produit-elle ici grâce à la projection d'une surface corporelle ? La clinique nous permet plus précisément d'évoquer un fond continu d'apparition de sensations en mouvement qui donne volume au corps. La notion de «voluminosité ${ }^{14}$ » proposée par Merleau-Ponty semblerait ici plus pertinente, dès lors qu'elle privilégie un espace à trois dimensions faisant surgir la présence : il s'agit selon Merleau-Ponty de faire parler l'espace et la lumière, à l'instant où surgit le geste. De plus, ce n'est pas une image du moi-corps qui se trouve projetée en une fabrique de lieu artistique, mais plutôt l'éclosion d'un volume corporel inconnu cerné en un champ de regard. Et ce volume corporel aurait pour caractéristique d'être habitable par tous ceux qui le pratiquent en passant.

Un autre moment important de la cure de l'analysante ici évoquée fut la possibilité de se séparer de sa fresque en demeurant dans le plaisir, sans retourner dans les limbes à la manière d'une ombre. Elle énonce à ce moment-là : " Je me suis séparée de la toile sans être écorchée, je la laisse aux autres. Ce n'est plus douloureux mais tranquille : c'est nouveau pour moi. »

Les processus d'engendrement d'objets inconnus et de corps étrangers ne définissent aucunement une structure psychique, mais ils déploient des procédés insolites de prise de corps et d'entrée dans un champ de regard. À partir de l'expérience de créateurs tels que Bram Van Velde, j'écrivais en

12.S. Freud, « Le moi et le ça » (1923), dans Essais de psychanalyse, Paris, Payot, 1981, p. 238.

13. S. Le Poulichet, 1996, op. cit.

14. " Une voluminosité qu'on exprime d'un mot en disant qu'une chose est là » note MerleauPonty, op. cit., p. 216. 
1996 que « l'objet créé prend alors la fonction d'un corps intime et étranger enfin investissable, qui représente un objet métaphorique pour le psychique lui-même ${ }^{15}$ ». Et j'ajouterai aujourd'hui, à partir de l'expérience des cures avec des créateurs, que l'expérience analytique permet aussi de fonder un lien et un lieu où se ressource et s'amplifie cette dynamique à travers un dispositif spéculaire transférentiel, de telle sorte que le rapport au danger, à l'angoisse, aux fantasmes et aux identifications s'en trouve véritablement transformé. Il faut ajouter que la relation à l'analyste constitue bien souvent pour ces analysants la première expérience de fiabilité et de continuité d'un lien où s'éprouve un nouveau mode d'articulation du corps à la parole et aux images.

Cela n'entraîne pas, contrairement à ce que laisse entendre un ancien préjugé, que la création artistique soit amenée à se tarir à cause de l'expérience analytique. La clinique montre au contraire qu'elle y trouve les sources et les motifs d'une plus grande liberté, tout en laissant place à d'autres manières de prendre corps et présence dans les liens aux autres, notamment à travers le sexuel. L'activité créatrice peut alors en bien des cas se révéler d'autant plus forte que se manifeste simultanément un investissement amoureux et sexuel.

Le surgissement au cours d'une analyse d'un nouveau champ de regard et de désir qui sécrète l'événement de la présence et de la "voluminosité » permet d'apprivoiser une " poussée poétique qui coupe le souffle depuis l'enfance », en transformant le rapport à un idéal inaccessible et en mettant en œuvre un heureux processus de séparation.

\section{BIBLIOGRAPHIE}

BECKETT, S. 1989. Le monde et le pantalon, Paris, Éd. Minuit.

FREUD, S. 1923. « Le moi et le ça », dans Essais de psychanalyse, Paris, Payot, 1981.

LACAN, J. 1949. "Le stade du miroir comme formateur de la fonction du Je », dans Écrits, Paris, Le Seuil, 1966.

LACAN, J. 1973. Le Séminaire, Livre XI, Les quatre concepts fondamentaux de la psychanalyse, Paris, Le Seuil.

Le Poulichet, S. 1996. L'art du danger. De la détresse à la création, Paris, Éd. Anthropos.

Le POULICHET, S. 2003. Psychanalyse de l'informe. Dépersonnalisations, addictions, traumatismes, Paris, Éd. Aubier/Flammarion.

Merleau-PONTY, M. 1961. «L'œil et l'esprit », Les temps modernes n 184-185.

15. S. Le Poulichet, 1996, op. cit., p. 8. 


\section{Résumé}

À partir d'expériences analytiques traversées par des créateurs, l'auteur interroge l'apport de ces derniers à la connaissance des processus psychiques. S'appuyant sur les questions des créateurs concernant le surgissement de la présence et la prise de corps, il s'agit d'élaborer les notions de champ de regard, de fabrique de lieu et de "voluminosité ».

C'est plus précisément à partir du trajet analytique d'une peintre que se trouve ici réinterrogée la fonction d'un dispositif spéculaire transférentiel dans la cure. Il apparaît alors que ce dispositif a le pouvoir d'amplifier la relance du désir qui sous-tend les processus créateurs ainsi que de transformer le rapport douloureux à un idéal inaccessible.

\section{Mots-clés}

Création, corps, regard, rêve, transfert.

THE FIELD OF VISION AND THE SURGE OF PRESENCE IN THE CREATIVE PROCESS

\section{Summary}

Working from analytical experiments undertaken with artists, the author investigates what these artists can tell us about psychic processes. Drawing on questions about the surging of presence and arrest, the article seeks to elaborate the notions of fiels of vision, space construction, and "voluminosity".

More specifically, it is by following the analysis of a painter, that we revisit the role of a model of mirror transference in treatment. It seems then that this model has the power to amplify the initiation of desire that underlies creative processes as well as transforming the painful relationship to an inacessible ideal.

Keywords

Creativity, body, gaze, dream, transference. 